\title{
BIM applied in historical building documentation and refurbishing
}

\author{
Hung-Ming Cheng ${ }^{\mathrm{a}}$, Wun-Bin Yang ${ }^{\mathrm{a}}$, Ya-Ning Yen $^{\mathrm{a}}$ \\ ${ }^{a}$ China University of Technology, 56 Sec. 3 ShingLong Rd., 116 Taipei, Taiwan \\ \{ hungmc, wunbin, alexyen\}@cute.edu.tw
}

KEY WORDS: BIM, architectural tectonics, heritage documentation, 3D laser scanning, time based documentation, heritage refurbishing

\begin{abstract}
:
Historical building conservation raises two important issues which are documentation and refurbishing. For the recording and documentation, we already have developed 3d laser scanner and such photogrammetry technology those represent a freeze object of virtual reality by digital documentation. On the other hand, the refurbished engineering of historic building is a challenge for conservation heritage which are not only reconstructing the damage part but also restoring tangible cultural heritage. 3D digital cultural heritage models has become a topic of great interest in recent years. One reason for this is the more widespread use of laser scanning and photogrammetry for recording cultural heritage sites. These technologies have made it possible to efficiently and accurately record complex structures remotely that would not have been possible with previous survey methods. In addition to these developments, digital information systems are evolving for the presentation, analysis and archival of heritage documentation.
\end{abstract}

\section{INTRODUCTION}

Monument and historic architecture have been considered as place and space of cultural heritage. As tangible cultural heritage, historical building reservation and reconstruction is one of the most significant challenges of cultural heritage.

For historical building reservation and reconstruction, survey and the acquisition of all possible data are the first step to initially concerning the objects and which will contribute to fundamental modeling for building recording and documentation.

These data not only include historic texts, archaeological figures, architectural information, but also administrative data and past drawings, sketches, photos etc. Moreover, these data also include current geometry information which can develop as multi-dimensional visualization with comprehensive, parametric databases to facilitate collaborative design and facility management.

In definition, BIM (Building information modeling) is an improved planning, design, construction, operation, and maintenance process using a metadata information model for building throughout its lifecycle.

Although the 3D digital construction and documentation of cultural heritage buildings involves a hybrid approach to visualization of several datasets such as 3D laser scanning, recording and survey techniques (photogrammetry) and CAD drawings. The most important process of BIM is to generate parametric model that needed to convert the surface of constructed facilities to the desired model. Then BIM system combines these parametric databases to facilitate collaborative design and facility management.

This research focuses on adapting data processing and geometrical recognition that utilizes $3 \mathrm{D}$ point cloud data towards the generation of a historic building tectonics. Tectonics refers not just to the "making the materially requisite construction to an art form". It represents multi-conscious attempts of architect and other historical meaning. Given the lack of appropriate construction documentation for many historic buildings, BIM generation may give a novel and important area of construction and technical research.
In this paper, we discuss two projects that explore an expanded role for BIM in the documentation and refurbishing of architectural heritage. These projects make survey and recording for preliminary experiments which are in scale, freeze models of virtual reality of digital documentation include: JingTong Train Station, located at the important railway of transporting coal; Taipei Xia-Hai City God temple, 17th century heritage building Located in Da-Dao Cheng, was one of the better-developed areas in Taipei. With these project, we proposed web-based BIM database for materials and methods of construction specific to represent heritage feature and tectonics. Historic Building Information Modeling (HBIM) is a special application of BIM, which is a new approach of utilizing parametric objects. The original digital data are built from historic data and system capturing for mapping the parametric objects onto a point cloud and photogrammetry data.

These new digital information systems allow for the production of multi-purpose models that can be used for more than just visualization. This paper presents a design framework for the integration of new developments in this area, Historic Building Information Modeling articulated with tectonics. HBIM provides a solution for parametric modeling of historic buildings/objects from laser scan or photogrammetric survey data. HBIM includes a plug in library of architectural objects for Building Information Modeling software platforms and a system for correlated with designing these objects (laser scan or photogrammetric survey data) and construction tectonics.

\section{HISTORICAL BUILDING MODELLING OF CONSTRUCTED FACILITIES}

The current approaches of historical building documentation and modeling are to collecting, organizing and integrating buildings' data into a single data structure is to model it using building information modeling (BIM) tools (Eastman et al., 2010). The parametric building models are generated by charactering building objects and the parametric relationships among them. These processes start by acquiring spatial data on site through surveying technologies, such as laser scanning and photogrammetry. The digitizing and documentation then are automatically registered into a 3D space and surface with targeted points for fine stitching. The points on the 3D surface 
are independently becoming an observation overall the data, identifying each object type, searching in a database of standardized objects, and adapting algorithms for optimal registration.

Historical building modeling for digitization and documentation is the process of capturing the spatial data and transforming it into a structured and parametric representation. These digital modeling and systems are generating useful information to designers, architects, constructors, owners, historical scholars and maintainers. For the project team, they may utilize and evaluate spatial modeling in order to solve complex problems such as design conflicts, quantity estimating, construction progress monitoring (3D simulation), project management (4D simulation), maintenance, during different phases of the building life cycle. For generating a 3D surface through remote sensing (3D laser scanning) and then identification, extraction and modeling of objects are the two main stages required to digitizing spatial modeling. (Goedert et al., 2003)

In 3D laser scanning, laser and sensors are used to capture the spatial features of the environment and generate a "point cloud" (Fig. 1). The conversion of point clouds into a surface that matches the real world is possible through employment of intelligent proximity algorithms and human intervention. The outcome of this conversion is a 3D surface that contains the spatial data of all elements presented in the scene. In the historical building modeling, Ranging (LiDAR) scanners based sensors and photogrammetry are two main methods for acquiring a building spatial information. Ranging (LiDAR) scanners based laser scanners, collect objects' coordination (x, $\mathrm{y}, \mathrm{z}$ ) and intensity (shape, silhouette and color) data to distinct points in a scene. A laser scanner returns data as a point cloud and visualization data through well-developed algorithm and software, which users interact and manipulate for construction model in a virtual environment (Jaselskis et al., 2003). The automatic manipulation and view data in a virtual environment is a unique feature of laser scanning and also are different from manual and traditional surveying.

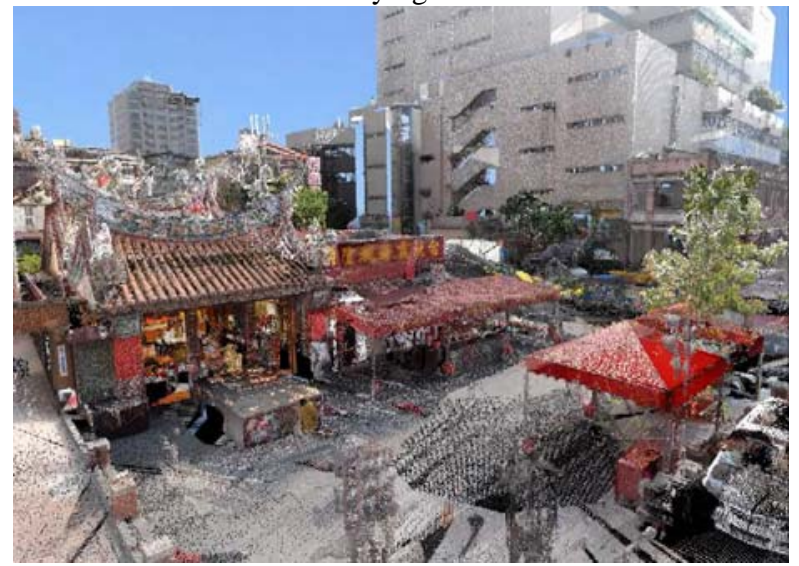

Figure 1. Point clouds of Taipei Xia-Hai City God temple

In this study, we exploit the issues of historical building preservation and documentation and explore an expanded role for BIM in the documentation and refurbishing of architectural heritage (Fig. 2). The experimental projects make survey and recording for preliminary experiments which are in scale, freeze models of virtual reality of digital documentation include: JingTong Train Station, located at the important railway of transporting coal; Taipei Xia-Hai City God temple, 17th century heritage building Located in Da-Dao Cheng, was one of the better-developed areas in Taipei. With these projects, we proposed web-based BIM database for materials and methods of construction specific to represent heritage feature and tectonics.

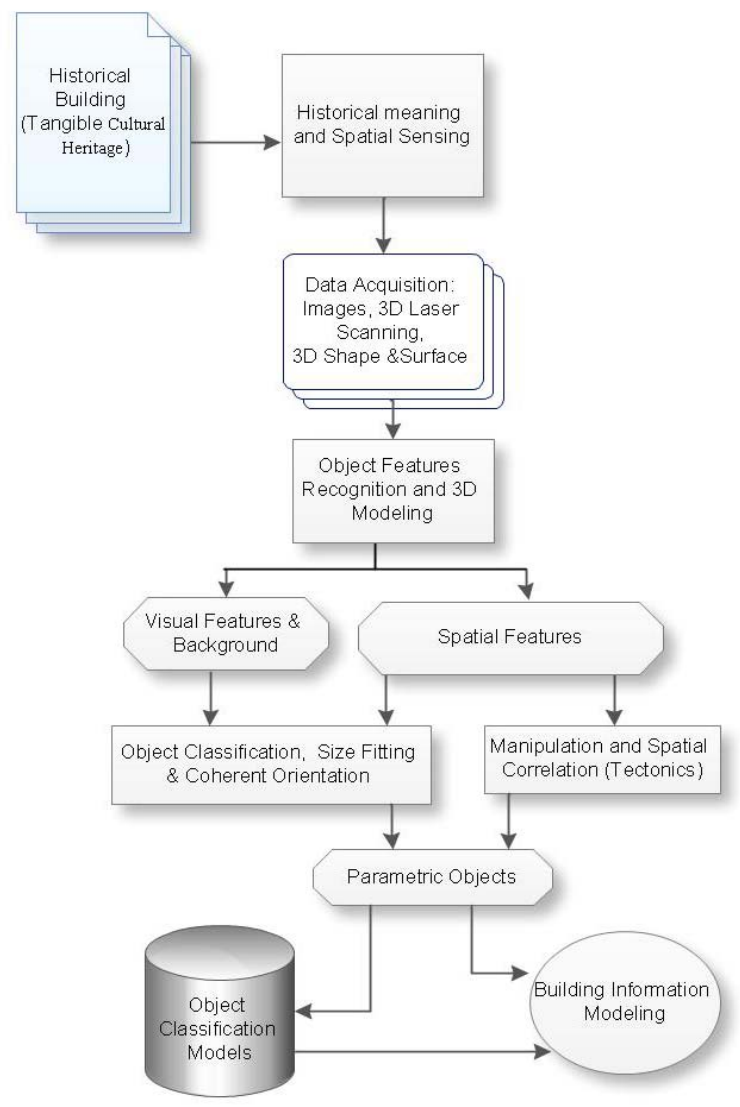

Figure 2. point cloud of Taipei Xia-Hai City God temple

\subsection{D data recognition and fitting}

The procedures of 3D data recognition and fitting involved several methods which process with middle-ware and algorithm. 3D data recognition in environmental and spatial modeling is the task of identifying the elements that are related to the construction of facilities. According to Brilakis and Soibelman (Brilakis et al., 2008), object recognition in environment and building structure is considered by characteristics of material images, which have low variability and opposite simulation. As recognition of objects, a general recognition methods (ie. face, fingerprint... etc.) cannot satisfy recognition of environment and building related applications. There are different methods developed for recognition of construction objects, which are material-based and shape-based. For 3D geometry data acquiring, these method are main process for recognizing for BIM system.

In material-based object recognition, features such as color, texture and structure are used to identify and classify object elements in images. Material-based recognition consists of three steps which are object representation, matching and classification. It initially decomposed each image into its basic features such as color, texture and structure. And by applying filtering techniques, surface material are cropped into regions using clustering methods to computing the material signatures of each cluster. Each cluster should then be identified and isolated by comparing signature with the feature signatures of the materials and vector and store material image. The last step is image classification, which basically classifies images into 
similar classes of objects, shapes or materials depending on the type of classification. By material-based recognition, the building and environmental material are extracted and stored in a knowledge-based system based on the material information (Brilakis et al., 2005).

Shape-based recognition is a complementary method which uses shapes and figures for identifying of linear and nonlinear building and environment with the image content. According to geometry recognition methods, shape recognition includes three stages, which are shape representation, shape matching and shape classification (Zaboli et al., 2008). There are different features that can be used to represent shape of a building such as the silhouette, the outlines, or the target points. One method that was implemented and tested for building related applications is mainly based on an outlines algorithm (Brilakis et al., 2008). This algorithms works by selecting image areas with similar shapes and a certain degree of uniformity, recognizing the shape characters that consist of each cluster, computing the maximum cluster dimension (MCD) and the maximum dimension along the perpendicular axis, and finally using these dimensions to evaluate the linearity and orientation of the "building spine" for each cluster (Brilakis et al., 2008). If the object is linear and its direction on the image plane can be computed based on the tangent of the MCD edge points. It can also be recognized if the object is a column or beam of building On the other hand, if the direction computed is with $45^{\circ}$ from the vertical or horizontal image axis relatively. There are techniques developed by Kwon et al. (Kwon et al, 2004) for fitting and matching which take advantage of the fact that most building elements can be fitted and matched using a few types of geometric shapes. Such as cubic, cylinders and planar surface, could be matched and recognized as elements such as columns, beams and walls.

\subsection{Parametric modelling and Object classification}

For Object Features Recognition is the next process which handles recognition of the objects' visible attributes using image processing tools. The input to this step is the rendered 3D surface that contains the correlated spatial and visual data of the structure to be modelled. The output is the augmented 3D surface containing recognized visual and spatial features of potential objects as well as the background. This process is concerned with the identification and selection of most appropriate visual and spatial features, selection of a suitable algorithm that most adequately represent the distinctive pattern of each feature, and finally, incorporating a novel method that can make feature representation invariant with respect to variations of viewpoint and illumination conditions. To satisfy these concerns, robustness of image analysis tools in recognition of information-rich features and selection of appropriate algorithm for feature detection and description that also handles invariance are critical. Recognition performance of this step is influenced by data accuracy and density of the input, which should be determined by validating the outcome of the previous steps.

The resulting model is a compact summarization of both the appearance and geometry information of the target. Unlike earlier attempts for 3D object categorization, our framework requires minimal supervision and provides the capacity to estimate an object's pose along with its class label. Because the object is represented as a linkage structure of parts, recognition is robust in the presence of partially occluded objects; even if one or multiple parts are occluded, other parts can still gather further evidence for the target. We can employ this framework for accurately detecting and recognizing objects from arbitrary poses and under arbitrary illumination conditions in a highly cluttered environment.

We use a category as an example database to better define this concept. As the observer moves on the viewing sphere the same part produces different appearances. The location on the viewing sphere where the part is viewed the most frontally gives rise to a canonical part.

\subsection{Building information modelling}

In definition, BIM (Building information modeling) is an improved planning, design, construction, operation, and maintenance process using a metadata information model for building throughout its lifecycle. Although the 3D digital construction and documentation of cultural heritage buildings involves a hybrid approach to visualization of several datasets such as 3D laser scanning, recording and survey techniques (photogrammetry) and CAD drawings. The most important process of BIM is to generate parametric model that needed to convert the surface of constructed facilities to the desired model. Then BIM system combines these parametric databases to facilitate collaborative design and facility management.

The specific phases of lifecycle of historical building include 3D modeling, time schedule with construction process, costrelated information and all aspects of life-cycle management. Designers can use BIM to explore alternative concepts, conduct value engineering and optimize their designs. Contractors can use a BIM model to 'rehearse' construction, coordinate drawings and prepare shop and fabrication drawings. On the construction process, they also can estimate time schedule with construction process and deal with the cost estimation and financial plan. Owners can use the model to optimize building maintenance, renovations and energy efficiency, as well as to monitor life cycle costs. BIM enables collaboration among designers, constructors and owners in ways the construction industry has never known before.

For monuments maintenance and repair work, BIM system has to input the data to the element database and quickly simulate the process of reconstruction or maintenance of the monument. BIM in historical building may exploit the relevant operating procedures, to monuments cultural assets reverse engineering operations. In order to facilitate consideration of the overall impact of environmental information and data access to the assessment of this study will integrate BIM system and environmental information, provide overall policy-makers to understand the overall planning and evaluation, rapid access to relevant information, the use of effective application management and planning, and through 3D navigation of forms, marketing monuments and cultural assets.

\subsection{Objective classification model vs. LOD}

In the procedure of BIM, level of development (LOD) is the specificity required for a particular element at a particular stage of the project. LOD identifies how much information is known about a building element at a given time. This "information richness" is requested by the processing stages on design and constructive project. As designers extract information from $\mathrm{BIM}$, the BIM system is regarded as a communication or collaboration tool. And the LOD framework on BIM is addressed some issues as below:

- During the design process, building systems and components progress from a vague conceptual idea to a precise description. In the past there has been no simple way to descript the model element level is moving along. The designers should know, but others often don't. 
- In a model though, a generic component placed approximately can look exactly the same as a specific component located precisely, and designers need something besides appearance to tell the difference. That is about tectonics articulation and infer the precision of the drawing not only from its appearance.

- It is possible to infer information from a BIM that the designer doesn't intend or unstated dimensions. This information can be exploited and developed with precision, assembly information before it's been finalized. Therefore, LOD specification allows the designer to clearly state the reliability of given model elements, so the concept becomes reliable.

- In a collaborative environment, where project team are more depending on information from the model in order to move their own work forward. It is necessary for the model users to know when information will be available to make their work forward.

Finally LOD Specification addresses these issues by providing an industry-developed standard to describe the state of development of various systems, assemblies, and components within a BIM. This standard enables effectiveness and efficiency in communication and execution by facilitating the detailed definition of BIM milestones and deliverables.

\section{PROJECTS AND CASES APPLICATION}

The experimental projects make survey and recording for preliminary experiments which are in scale, freeze models of virtual reality of digital documentation include: Jing-Tong Train Station, located at the important railway of transporting coal; Taipei Xia-Hai City God temple, 17th century heritage building Located in Da-Dao Cheng, was one of the betterdeveloped areas in Taipei. With these projects, we proposed web-based BIM database for materials and methods of construction specific to represent heritage feature and tectonics.

\subsection{Survey Planning and Scan Works}

For acquiring the 3D data of real environments, an integrate scanning work has to planning for the whole archaeological objects from several different scanner stations which can combine a set of order of multiple view (scanning worlds). The major steps in combining multiple views are survey planning and accurate registration that related to information management in multiple views to be combined successfully. Such set of information are organized as token ring or star topology, and captured by multiple views in serial survey with registration targets. (Fig.3)

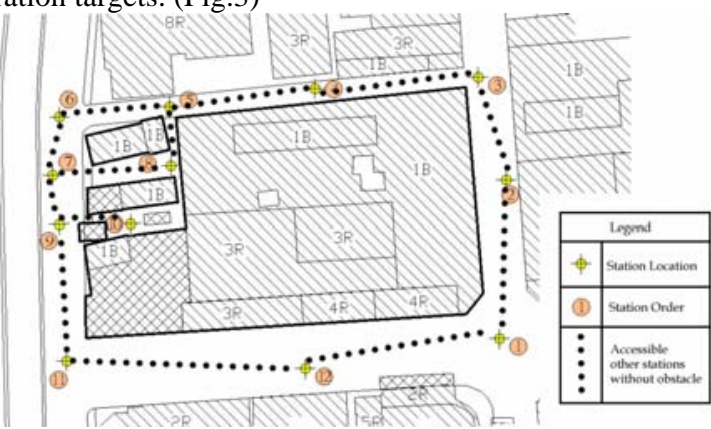

Figure 3. Survey Planning for Scanner Station

For development of 3D scanner supports 360 degrees field-ofview, the scanner has to move around archaeological objects to complete exterior scan views with registration targets. The laser scanner is difficult to work at those buildings placed closely without appropriate station-locations on scanning and registration entire surroundings. Therefore, several higher locations are selected nearby those buildings. Moreover, higher scanning locations are used to avoid obstructions problems and reduce numbers of scanning station. (Fig. 4). These obstructions accessing stations are manipulated by special devices which can raise 3D scanner machine for survey higher and wider. Mobile vehicle carries lift-able device and work with more function controlled.
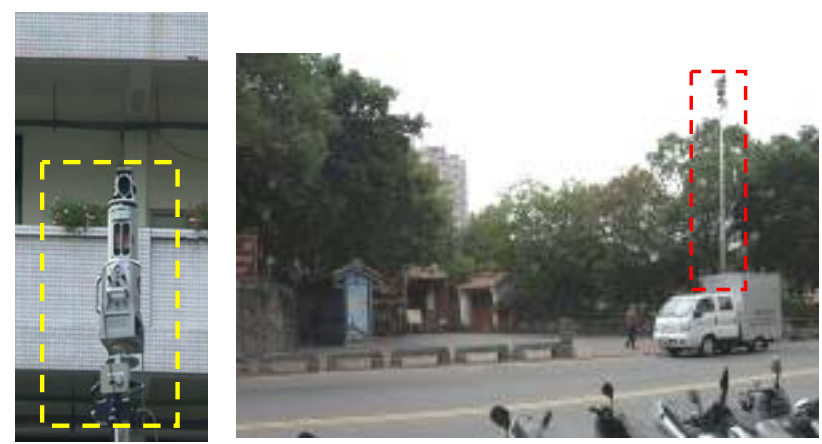

Figure 4.The device raising 3D scanner and working on vehicle

For those objects to be scanned in a well-controlled environment (for example: indoor space and no obstruction problem), objects can be oriented scan completely without extra registration targets. However, ranged scanning is often incomplete for obstructions or lack of controlled-high-view scans at outdoor site. There is no way to solve such as problems until a lift-able car and movable support are made up.

The other devices provide carrier of 3D laser scanner with liftable and stable character. It is not only fixing on the ground but also avoid to vibration. Furthermore, it can be controlled in height by expert experience for the best survey. The device with platform on the top can fixed scanner on the lift-pole. The each of multi-supported legs implements dynamic device for lift-up the truck that is for stable survey implementation without shaking by rubber tires. The lift-able device includes scissorslike instrument and platform, which includes a space for 3d laser scanner instrument and multi-action kinematics extending the range of survey and reach proper height for advantage process. Therefore, the creative work includes lift-able and stable character that is not only stability of scanner and also convenient adjusting height for the best survey efficiently. (Fig. 5.)

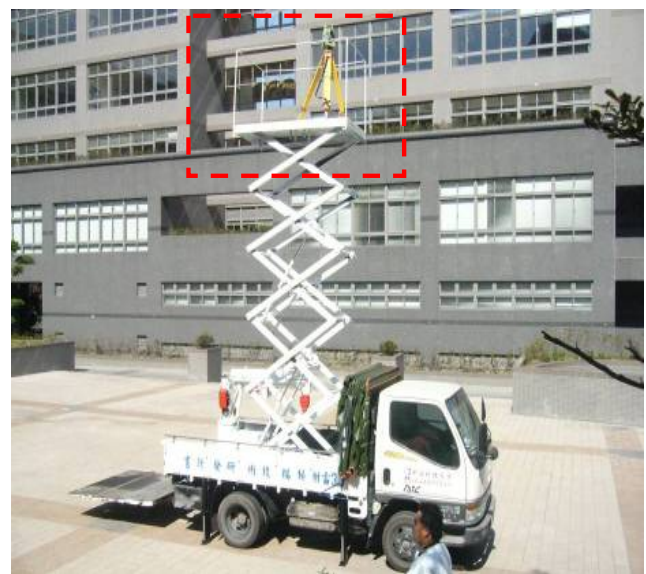

Figure 5. lift-able car and movable support 


\subsection{Documentation and Monitoring of Cultural Heritage}

Virtual preservation takes advantage of the expansion and longterm saving. Cultural heritage needs these data for sustainable monitoring. In particular, the heritages in real environment are irreversibly damaged by environmental disaster or atmospheric damages. Those damages sometime were discovered too late. High accuracy 3D scanning, at regular times, could detect deformations and cracks. These data of monitoring are the fundamental knowledge for reconstructing heritage.

The 3D documentation archives the spatial data of cultural heritage. Documentation should be considered as an integral part of a greater action in general documentation of the cultural heritage. These as-built and spatial data include columns, beams, walls, roof and more other geometrical shape, texture and colour. The as-built and environmental data with historical documentation, architectural documentation store as the whole picture of cultural heritage. For the historical preservation, digital archive of high quality three-dimensional models would give improvement in the restoration science field. Digital archives thus can be used as reference for monitoring and restoration of cultural heritages.

In Jing-Tong train station, we got the several point clouds (Fig. 6) from survey stations and registrate these 3D data for as-built and environmental drawing documentation (Fig. 7).

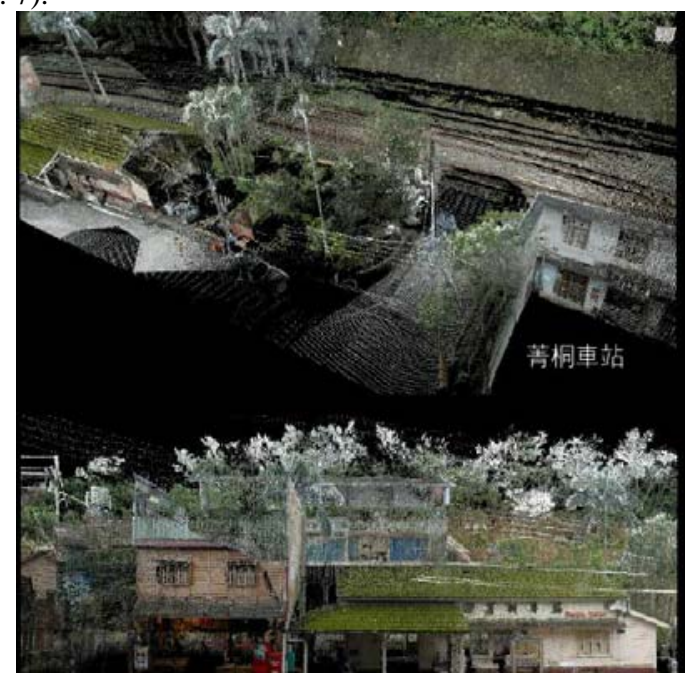

Figure 6. Point clouds of Jing-Tong Train Station

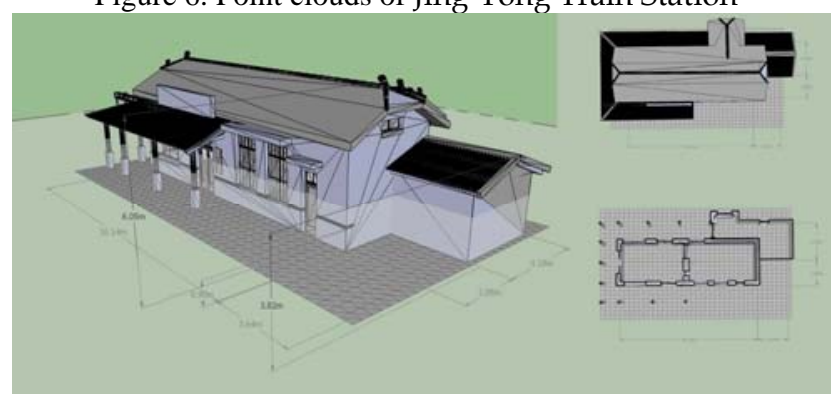

Figure 7. 3D Documentation (Jing-Tong Train Station)

\subsection{Taipei Xia-Hai City God temple}

The temple was built in 1856, located in Da-Dao Cheng, a port city that was one of the well-developed commercial areas in Taiwan. The belief meaning of Xia-Hai City God temple is ensuring prosperity and peace through its blessings. Nowadays young people come to pray for a happy love life and good job. Every year the temple holds the annual "City God Birthday Parade" on May 13th, a celebration that is classified as an intangible asset by Taipei City government.

During the first phase of the environmental investigation was spent to set properly the building type of understanding that will be followed in the future of the project, beginning to develop the 3D data acquisition and parametric model. More experiments are needed to fulfill the 3D model in specific applications, deepening the interoperability issue, arriving to define guidelines for the correct use of BIM for the refurbishment of the existing buildings. Existing building have a number of major difficulties than new buildings, but the use of new technologies mentioned above gives the opportunity for professionals to manage data, querying the 3D model (Fig. 8) through a iterative and interactive steps that characterize the BIM process.
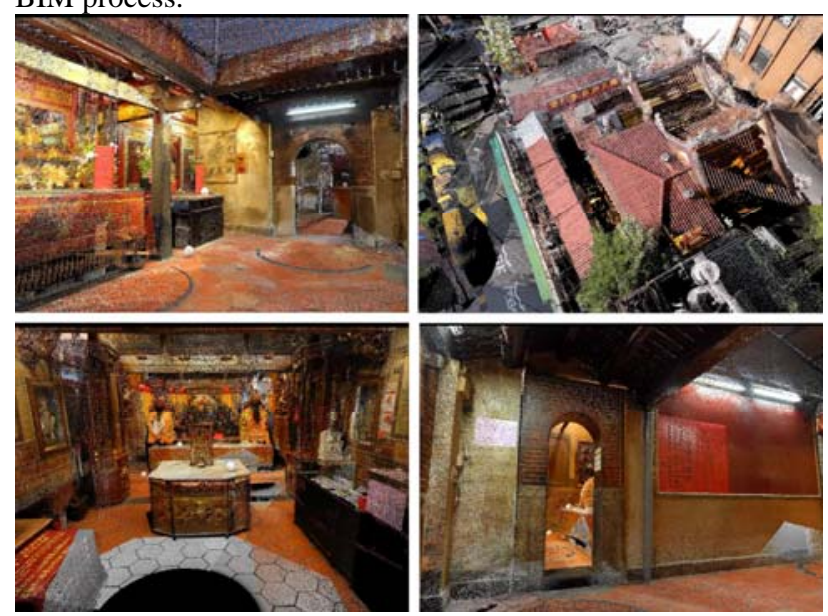

Figure 8. Point Clouds of Xia-Hai City God temple

On the other hand, tectonics in architecture is usually defined as "the art or science of construction", "a poetic of construction and design". tectonics refers not just to the "making the materially requisite construction to an art form". It is transcending the banality of mere building by the modelling of the physical thing that reveals a conscious attempt by the designers and architects to make information.

The Xia-Hai City God temple is on the refurbishing project. It needs renovation of roof and plafond of central temple. The temple also need to record and documentation that can be exploited in the design phase for natural building. The roof structure will correct and change its native structure because some part is missing and not right for the original design. Based on necessary of tectonics and BIM, the project is organized and developed considering three essential elements:

- The working methodology based on the data exchange following the interoperable way and the problems solving related to it;

- The data hierarchy during the different phases of the design and the role of professionals in the filling's data steps in the 3D model and BIM (LOD); 
In order to organize the work to be performed, possible areas concerning the project management, in which it is necessary share information to develop the full BIM, have been identified. The geometric data capture needed for the developing of the parametric model in Autodesk Revit. This step took place in two parallel and complementary ways: the first way was characterized by a topographic survey using a total station for the measurement of the essential elements of the external facades, while the second by the use of laser scanner for the external and internal part of the temple building.(fig. 9)
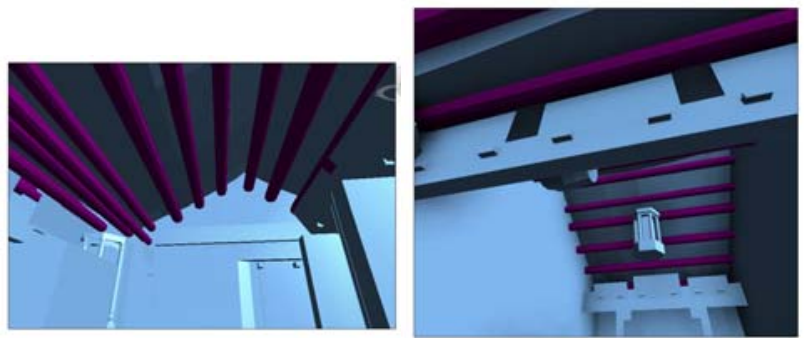

Figure 8. Autodesk Revit on Roof of Xia-Hai City God temple

\section{CONCLUDSIONS AND FURTHER WORKS}

Having access to an as-built historical monument and environment of an existing building can enhance project planning, improve data management, support decision makings and increase the productivity, profitability and accuracy of a project in construction industry. However, current building modelling methods for existing buildings rely heavily on human manual to survey and generate logical building 3D model and the parametric relationships between them. 3D data acquisition can be collected automatically using laser scanners, but digital interpretation of point clouds, stitching and object fitting are all performed manually. This process is time-consuming and costly, which reduces the benefits of historical building modelling and refurbishing projects.

This paper raise the integration and employment of 'Level Of Development(LOD)' methods and tectonics components that can support a wide range of as-built buildings, thus achieving automation of the digital modelling processes involved in the generation of HBIM. Experimental implementation of this research is expected to significantly reduce the time and resources needed to model a structure, making as-built modelling affordable for even smaller architectural projects. This can focus on full life-cycle modelling in the historical architecture and monuments and facilities management.

Historical building refurbishing involves a lot of renovation and expansion of existing facilities which needs automatically compiled parametric as-built models would enable designers to use BIM tools without rebuilding the existing structures. The implementation of current projects still have many challenges that require research at the intersection of BIM, visual and spatial sensing and sensor systems, computer vision and image processing technologies. These projects sometimes are aggravated by the need to deploy and utilize the expected prototype in outdoor environments. The main challenges involved in this investigation is the integration of video capture and laser scanning technologies to capture not only the precise geometry but also generating semantically meaningful components of structures such as columns, roofs, architectural decorations, plafonds and other prototypes of historical architecture. Another challenge is to create (development) a set of parametric building objects to represent the components, including their behavior, their connections and the relationships between them such as LOD in BIM. The goal of this paper is to provide a complex models that can be imported into the original or prime object forms of BIM tools so that they can be manipulated for building elements of tectonics and readily transferred to level of development(LOD) which are defining at the assembly ,component level and the reliability of information. There are also structural analysis, material quantity take-off, energy simulations and other analyses in BIM..

\section{REFERENCES}

Brilakis I., Soibelman L., Shinagawa Y., 2005, Material-based construction site image retrieval, Journal of Computing in Civil Engineering, American Society of Civil Engineers 19 (4), pp. 341-355.

Brilakis I., Soibelman L., 2008, Shape-based retrieval of construction site photographs, Journal of Computing in Civil Engineering, American Society of Civil Engineers 22 (1), pp.420.

Brilakis I., Lourakis M., Sacks R., Savarese S., Christodoulou S., Teizer J., and Makhmalbaf A., 2010. Toward automated generation of parametric BIMs based on hybrid video and laser scanning data. Advanced Engineering Informatics Vol. 40(4), pp.456-465.

Del Giudice, M., \& Osello, A., 2013. Bim for Cultural Heritage. International Archives of the Photogrammetry, Remote Sensing and Spatial Information Sciences, 1(2), pp.225-229.

Eastman, C., 2010. BIM Handbook: A Guide to Building Information Modeling. John Wiley and Sons, Inc. Hoboken, New Jersey

Goedert J., Bonsell J., Samura F., 2005, Integrating laser scanning and rapid prototyping to enhance construction modelling, Journal of Architectural Engineering 11 (2), pp.7174.

Jaselskis E., Gao Z., 2003, Pilot study on laser scanning technology for transportation projects, in: Proceedings of 2003 Mid-continent Transportation Research Symposium, Ames, Iowa.

Kwon S.W. , Bosche F., Kim C., Haas C.T., Liapi K.A., 2004, Fitting range data to primitives for rapid local 3D modeling using sparse range point clouds, Automation in Construction 13 (1), pp.67-81.

Penttilä, H., Rajala, M., Freese, S., 2007. Building Information Modelling of Modern Historic Buildings, Predicting the Future, 25th eCAADe Conference Proceedings , pp.607-613

Russell, Peter; Elger, Dietrich, 2008, The Meaning of BIM, Architecture in Computro, Proceedings of the 26th eCAADe Conference, Antwerpen, pp.531-536.

Xiong, X., Adan, A., Akinci, B., \& Huber, D., 2013. Automatic creation of semantically rich 3D building models from laser scanner data. Automation in Construction, 31, pp.325-337.

Zaboli H., Rahmati M., Mirzaei A., 2008, Shape recognition by clustering and matching of skeletons, Journal of Computers 3 (5), pp. 24-33.

Revised January 2015 